\title{
THIRD INTERNATIONAL SYMPOSIUM ON ARTIFICIAL INTELLIGENCE AND THE GAME OF CHESS
}

\author{
B. Pernici \\ CSISEI-CNR - Politecnico di Milano \\ M. Somalvico \\ Milan Polytechnic Artificial Intelligence Project \\ Dipartimento di Elletronica
}

The Third International Symposium on 'Artificial Intelligence and the Game of Chess', organized by the Milan Polytechnic Artificial Intelligence Project, Politecnico di Milano, and the Italian Chess Federation, was held in Milan on March 14-15, 1986.

Nine papers by authors coming from Italy and from five other European countries were presented at the Symposium; the topics covered ranged from chess databases to knowledge representation in chess and problem solving.

The participation to the Symposium was of about fifty researchers and chess-players interested in computer chess, from Italy and abroad. Discussion was lively during the presentations and on the panel at the end of the Symposium. The Proceedings of the Symposium of about two hundred pages have been distributed.

The principal new topic, with respect to the previous Symposia held in Milan, was that of chess databases. Databases are being used to analyze exhaustively chess endgames, since complete analysis can be performed starting backward from final positions. This type of analysis has allowed to determine the minimum correct sequence of moves from any position of several particular endgames, and in some cases with the use of this method it has been proven that endgame theory concerning the assessment of positions and the estimated number of optimal moves to win, starting from any position of a particular type of endgame, has in some cases been incorrect.

However, such databases are on one hand very expensive to build in complex cases, on the other hand they do not provide solutions to human players, since they do not provide rules to perform a correct sequence of moves. It has also been shown that extracting rules from a database (either automatically or by a human player) is a very hard task. The solution proposed by Dr. Van den Herik and Dr. Herschberg of Delft University, The Netherlands, is one of extracting patterns from endgame databases.

Several types of endgame have been compiled as ORACLE databases by Mr. Roycorft, Editor of the EG Journal; in his paper it was also suggested that chess databases are a good topic of investigation for programmers, $\mathrm{AI}$ researchers, psychologists, and chess-players.

As in the case of endgames, representing complex knowledge is a very important aspect for all phases of the game. Prof. Pitrat of the French National Research Council presented a paper based on the concept that positions should not be evaluated separately, but instead other kinds of information should be used in chess-playing programs, such as sophisiticated evaluation of moves. This kind of information is needed for devising strategic plans: it may be necessary to reach a position which is evaluated with a lower score than the starting one to eventually reach a particular strategic goal.

The problem of using plans was examined also by Dr. Armenise and Prof. Enriquez of the University of Bari, in the framework of finding solutions to tactical positions by means of plans. Plans are constructed using motivations, subobjectives, confutations of different plans, which are combined to propose moves and reduce search. 
Prof. Seidel of the Technical University of Berlin examined a notation to represent chess positions semantically. Syntactic representation of sequences of positions just in terms of moves is not sufficient. Semantic units have been defined to describe positions, such as 'bottom square', 'diagonally adjacent squares', 'attacked by', 'protected by', and are connected in semantic nets. Effects of moves can also be classified in syntactic (threatening, defending) and semantic effects (e.g. creation of threat, counteraction). Knowledge about how to accomplish moves' effects is required, and it should be possible to combine such knowledge to introduce further knowledge arriving at move effects which closely meet the terms used in ordinary chess language. Such knowledge can then be used to generate plans to guide the search process.

A third group of papers was about chess problem solving. Mr. M. Guida from Politecnico di Milano presented an expert system for the analysis of two-moves endgames, able to recognize the idea the composer wanted to show, starting from the complete solution of a chess problem.

Dr. Lindner from Budapest presented the results obtained by problem solving programs, comparing the performances of programs on a problem set. The high standard attained by these programs is of great significance to the chess-problem world, as both the productivity of problem composers can be multiplied and the publication of defective problems can be completely avoided. Requirements of problem solving programs were also discussed.

Dr. Fiorentini, Dr. Dara and Mr. Ferrari presented in two papers results obtained within the Kyberchess project of the University of Milan. The first paper was about an interface, GATHERER, that presents the results of the complete analysis of a problem in compact way, similar to that adopted by humans. The second one was about the use of a parallel architecture to evaluate moves' legality in an efficient way, in particular in complex situations.

A panel was held with the participation of Dr. Van den Herik, Prof. Pitrat, Prof. Somalvico, and Dr. Eng. Pernici. The topics discussed included the role of computer science in general and of AI in particular in the game of chess, which aspects in chess games are suitable for experiments in AI, about the results obtained to date in the field and expectations for the future.

In conclusion we can assess that this Symposium, having been held for the third time, has established itself as a known reference for those scholars who are interested in AI-based researches aimed to study and emulate, with AI machines, human activities such as appear in playing chess; these are logical and analytical, though nonetheless creative.

The next Symposium is scheduled for Spring 1988. In Summer of 1987, during IJCAI, which will be held in Milan from August 23 to August 29, a special event is scheduled on computer chess.

Copies of the Proceedings may be requested from:

\author{
Mr. Nicolà Palladino \\ Viale Caldara 13 \\ I-20122 Milano \\ Italy
}

